\title{
Aided 3D Facial Restoration for Plastic Surgery Planning using Elastic Radial Curves
}

\author{
Hermessi Haithem \\ Dept. of Software Engineering and Information \\ Systems \\ Higher Institute of Computer Science \\ Ariana, Tunisia
}

\author{
Mourali Olfa \\ Dept. of Software Engineering and Information \\ Systems \\ Higher Institute of Computer Science \\ Ariana, Tunisia
}

\begin{abstract}
Improving appearance and guarantying healthcare are the important objectives of facial plastic surgery. Face can be the seat of tumors that can be restored using 3D images in a way to surpass $2 \mathrm{D}$ limits such as change in illumination conditions and pose variation. This work proposes a Riemannian geometry approach to illustrate the degradation of facial tumefaction which is based on geodesic paths between healthy and swollen half of the face. The progressivity of tumor volume assists plastic surgeons in approaching the harmonious shapes for facial restoration. We introduce a preprocessing step to prepare 3D scans chosen from FRAV 3D database then sculpted to simulate unilateral tumors. We present facial surfaces by indexed collections of radial curves emanating from the nose tips. Symmetric indexed radial curves are compared using elastic shape analysis.
\end{abstract}

\section{General Terms}

Elastic 3D face analysis for plastic surgery planning.

\section{Keywords}

Riemannian geometry, symmetric radial curves, geodesic path, 3D facial restoration, plastic surgery planning.

\section{INTRODUCTION}

Computer aided plastic surgery has actively been researched in recent years, and various techniques using ideas from 2D image analysis have been presented. The range of facial malformations that plastic surgeons are asked to alter is wide. Despite a significant progress has been made, automated 2D image-based surgery planning still facing multiples limits such as illumination and pose changes. Recently, the explosion of 3D imaging tools as a research field has made an effort to assist surgeons in achieving optimal functional and esthetic results. Computer- aided surgery can be divided into 4 phases: data acquisition phase, planning phase, surgical phase and assessment phase [1]. During the planning phase, it allows surgeons to analyze patients by performing 3D measurements and to manipulate deformed or missing anatomy. Computer aided surgical planning has been investigated by many researchers in the past decade [1,2,3,4,24,25]. Bottino et al [2] proposed a computer-aided technique for planning plastic surgery. Their quantitative approach is able to automatically suggest effective patient specific improvements of facial attractiveness. In order to normalize data, 3D faces are aligned in 3D space by translating their centroid in the origin and making their symmetry axes coincident with the reference axes. In the same research field, Bhandarkar et al [4] presented an algorithm that reduces the fractures and reconstructs the craniofacial skeleton. Their approach is based on surface matching of $2 \mathrm{D}$ gray scale images.
3D face reconstruction and surgical planning can help surgeons to reduce the potential of risks in plastic surgery. There have been several researches proposed $[5,6]$. Lee et al [5] proposed to generate 3D facial models and simulate surgical operations using a metamorphosis technique. Their algorithm extracts a set of contours corresponding to facial surface. Then, it links every two consecutive contours to approximate the facial model.

Also, the task of 3D facial restoration has been approached in the literature using the geometry aspect of the face. Drira et al [7] proposed a geometric framework for comparing and matching 3D face shapes. Their approach is based on Riemannian analysis that utilizes an elastic metric for measuring deformation due to facial expressions. In the same context, elastic shape analysis was presented in [8] to restore 3D faces under occlusions for recognition purposes. Facial surfaces are approached by indexed collections of radial curves. Occlusions detection and restoration of partially occluded faces was also approached by Colombo et al $[9,10,11]$. The detection method considers occlusions as local deformations of the face and the restoration task is based on Principal Component Analysis (PCA) reconstruction. Furthermore, the task of $3 \mathrm{D}$ facial reconstruction was approached in the literature [20,21]. Levine et al [20] discussed the state of the art of 3D facial reconstruction methods which attempt to reconstruct 3D facial models of individuals from their $2 \mathrm{D}$ images.

The rest of the paper is organized as follows: In Sect. 2, we present a series of scan pre-processing tasks that are undertaken before comparing symmetric faces halves. Then, we expose the facial surfaces representation and the mathematical model of curves comparison. Experimental results on the swollen faces database are discussed in sect. 3 . Finally, conclusions and future research directions are outlined in Sect. 4

\section{METHODOLOGY\& CONTRIBUTION}

This work is based on a Riemannian framework for 3D facial shape analysis. Scans are firstly chosen to be frontal. Then we proceed to fill holes due to acquisition. This framework is based on elastically matching and comparing of radial curves emanating from the nose tip. The extracted curves are symmetrically compared to illustrate the degradation of the unilateral tumefactions localized in one of the face halves. The main contributions of this work are:

- Exploitation of facial symmetry by comparing the shape of radial curves in the healthy half with those of swollen half of the face.

- Adoption of the Riemannian shape analysis of facial radial curves in $3 \mathrm{D}$ facial restoration. 
- Construction of a swollen 3D faces database.

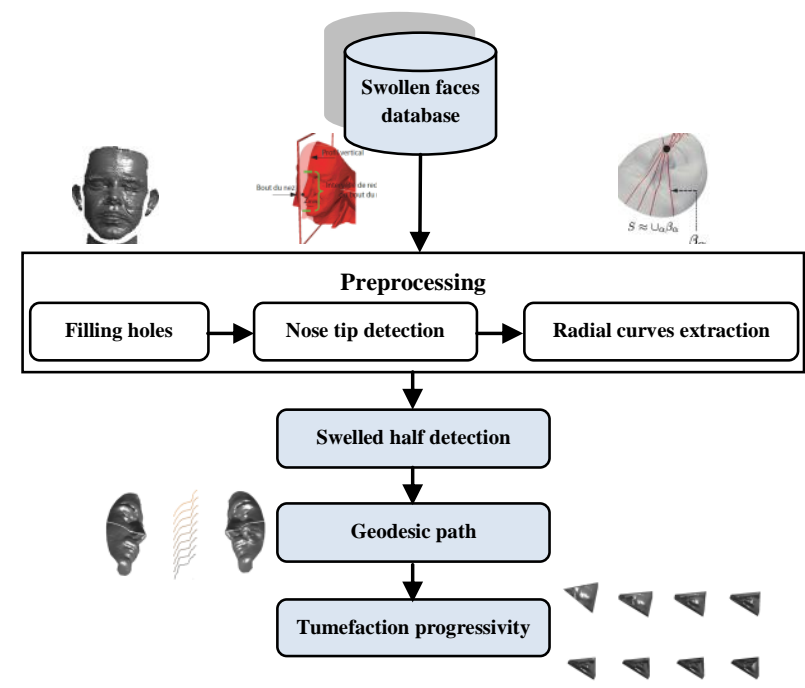

Fig. 1. Overview of the proposed method.

\subsection{Aided 3D facial restoration for plastic surgery planning}

Our proposed method for aided facial restoration is illustrated in figure 1 . In the following, we will expose the tasks with more details.

\subsubsection{Scans sculpting}

Not many 3D face databases exist, and they are essentially built to support face recognition research. In addition, to the best of our knowledge, no database rated for 3D swollen faces exists. During this work, we have simulated local deformation in face cheeks using blender, a toolbox for design and animation of 3D scans. We have selected neutral scans from FRAV 3D then sculpted them locally in the cheeks. We suppose that tumors are unilaterally.

\subsubsection{Scan preprocessing}

As illustrated in figure 1, our proposed method starts by processing the input scan suffering from cheek tumefaction in order to restore the harmonious shape of the face. Images present some imperfections as holes and include some undesired parts unnecessary for the restoration (hair, neck, ear, etc.). Therefore, we introduce a pipeline of 3D mesh processing filters of the literature [7,12]. First, our approach begins with filling holes due to acquisition using Delaunay triangulation. Then, the nose tip is automatically localized in frontal scans by a first transverse plane slicing the facial surface and resulting in a horizontal profile. The second intersection is sagittal passing through the maximum of the obtained horizontal profile. The minimum depth on this curve is interpreted as the nose tip. Finally, a sphere function having center the nose tip and radius $\mathrm{R}=100 \mathrm{~mm}$ is constructed to limit the essential part for the face and the part outside the sphere is cropped [13]. Figure 2 illustrated the result of the preprocessing step.
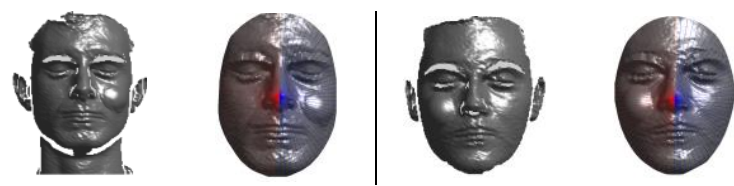

Fig. 2. Examples of preprocessed swollen faces.

\subsubsection{Radial curves extraction}

After preparing the scan, we consider $\mathrm{S}$ the facial surface denoting the output of the previous preprocessing step. We suppose that it is a continuous surface [7]. $\beta \alpha$ is a radial curve on $\mathrm{S}$ which makes an angle $\alpha$ with the vertical curve chosen to be the reference on frontal scan. Each radial curve is the result of slicing the facial surface by a plane $\mathrm{P} \alpha$ that has the nose tip as its origin and makes an angle $\alpha$ with the vertical plan. $\beta \alpha$ is the intersection of $\mathrm{P} \alpha$ and $\mathrm{S}$. the collection of indexed radial curves is extracted by repeating this step at equal angular separation. $\mathrm{S}$ can be approximately reconstructed from these radial curves according to $\mathrm{S} \approx \mathrm{U}_{\alpha} \beta_{\alpha}=\mathrm{U}_{\alpha}\left\{\mathrm{S} \cap \mathrm{P}_{\alpha}\right\}$. This procedure allows to capture the shape of the face according to $[7,12]$ and forms the mathematical representation of that surface. It's also an efficient tool for analyzing elastic shapes of curves and modeling deformation associated to face tumefaction that will be diagnosed by plastic surgery specialist which is the main goal of our work.

\subsubsection{Facial Symmetry exploitation}

Human face is bilaterally symmetric but not perfectly due to nature. Facial symmetry changes from person to another and was exploited in literature. Chen et al [14] exploit an automatically detection technique of symmetric axis for face recognition purposes. In the same context, Harguess et al [15] tried to find a relationship between face symmetry and face recognition according to symmetry scores. They admit that face recognition with eigenfaces using the average half face is significantly higher than using the full face in all subgroups regardless of symmetry score. Facial symmetry was also exploited in plastic surgery in [16]. Azinfar et al [16] present a method for specifying the facial symmetric points for Botox injection when used for cosmetic purposes.

In this work, we have adopted facial symmetry to compare the swollen half of the face with the healthy one. Since the curves are indexed, each radial curve of the swollen half has its symmetrical one in the healthy half of the face. This method allows us to compare symmetric curves according to the vertical to illustrate the geodesic path between them in a way to show the gradual swelling of the area presenting a tumor.

\subsection{Riemannian analysis of symmetric elastic radial curves}

As mentioned earlier, our goal is to analyze shapes of facial surfaces. In other words, the shape of indexed symmetrical curves of the face will be studied based on differential geometric approach. In the following section, we will describe curves representation in $\mathbb{R}^{3}$. Then, we will follow the theory laid out by Joshi et al [17] for elastic shape analysis of open curves in $\mathbb{R}^{\mathrm{n}}$ which was particularized for facial curves in $[7,8,12,13,23,26]$.

\subsubsection{Curves representation}

We begin by considering a continuous open curve $\beta$ in $\mathbb{R}^{3}$. Similarly adopted in $[7,12,13], \beta: I \rightarrow \mathbb{R}^{3}$, for $I=[0,1]$, represent a parameterized curve on the face, We represent mathematically $\beta$ to analyze its shape using the square root velocity function (SRVF) denoted by $\mathrm{q}(\mathrm{t})$ according to $[7,12,13]$ :

$$
q(t) \doteq \frac{\dot{\beta}(t)}{\sqrt{\|\dot{\beta}(t)\|}}
$$

$q(t)$ is a special function that captures the shape of $\beta$ and it is particularly convenient for comparison of local facial shapes 
analysis in the presence of elastic deformations. Also, it simplifies computations under elastic metric [19]. We assume that the parameterization is non-singular, i.e. $\|\dot{\beta}(t)\| \neq 0$ for all $t$. The length of $\beta$ is the squared $\mathbb{L}^{2}$-norm of $q$ given by [7,12]:

$$
\|q\|^{2}=\int_{0}^{1}\left\langle q_{1}, q_{2}\right\rangle d t=\int_{0}^{1}\|\dot{\beta}(t)\| d t
$$

We denote by $\mathcal{C}$ (equat. 3 ), the set of all radial curves in $\mathbb{R}^{3}$ each represented by its SRVF. $\mathcal{C}$ becomes a Riemannian manifold with $\mathbb{L}^{2}$ metric on its tangent space. Due to the unit $\mathbb{L}^{2}$-norm, $\mathcal{C}$ is a hypersphere in the Hilbert space $\mathbb{L}^{2}\left(\mathrm{I}, \mathbb{R}^{3}\right)$ $[7,12,13]$.

$$
\mathcal{C}=\left\{\mathrm{q}: I \rightarrow \mathbb{R}^{3} \mid\|q\|=1\right\} \subset \mathbb{L}^{2}\left(I, \mathbb{R}^{3}\right),
$$

To illustrate the progressivity of the face tumefaction, we are conducted to compare the shape of two symmetric radial curves by computing the distance between them in $\mathcal{C}$ under the chosen metric. The shortest geodesic connection between two points in $\mathcal{C}$ is the distance between two parameterized curves of the face given by $[7,12,13]$ :

$$
d_{c}\left(q_{1}, q_{2}\right)=\cos ^{-1}\left(\left\langle q_{1}, q_{2}\right\rangle\right) .
$$

\subsubsection{Symmetric radial curves comparisons}

The framework presented by Joshi et al [17] allows computing geodesic paths that denotes optimal deformations between symmetric indexed curves. Therefore, these deformations are combined to obtain progressivity of cheek tumefaction. The geodesic path for $\alpha:[0,1] \rightarrow \mathcal{C}$ is calculated according to the given equation:

$$
\alpha(\tau)=\frac{1}{\sin (\theta)}\left(\sin ((1-\tau) \theta) q_{1}+\sin (\theta \tau) q_{2}\right),
$$

$\theta=d_{c}\left(q_{1}, q_{2}\right)$ denotes the angle between two parameterized symmetric curves $\mathrm{q}_{1}$ and $\mathrm{q}_{2}$ which is the geodesic distance between them. Figure 3 shows the geodesic path that illustrates the optimal deformation of the curve localized in the healthy half. This illustration can be helpful for computer aided reconstructive surgery. Indeed, the progressivity of a tumor volume assists plastic surgeons to approximate the normal appearance and to approach the harmonious shape of the face.
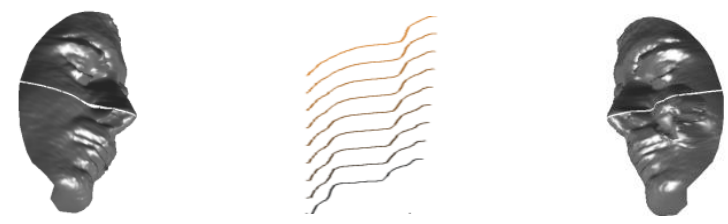

Fig. 3. Geodesic path between two symmetrical radial curves.

Figure 4 shows matching of two symmetric radial curves, one is located in the healthy half and the other in the swollen. In order to analyze the shape deformation of curves, we should identify the rotation group $\mathrm{SO}(3)$ and re-parameterizations group $\Gamma$ as an equivalence class in $\mathcal{C}[7,12,18]$. The elements of $\Gamma$ are viewed as re-parameterization function. Here, the equivalent class of $\mathrm{q}$ is given by: $[\mathrm{q}]=\{\sqrt{\gamma \dot{\gamma(\mathrm{t})}} \mathrm{Oq}(\gamma(\mathrm{t})) \mid 0 \in \operatorname{so}(3), \gamma \in \Gamma\}[7,12] . \quad$ The optimal matching of 3D symmetric radial curves is calculated using dynamic programming $[7,12]$ as given in equation 6 .
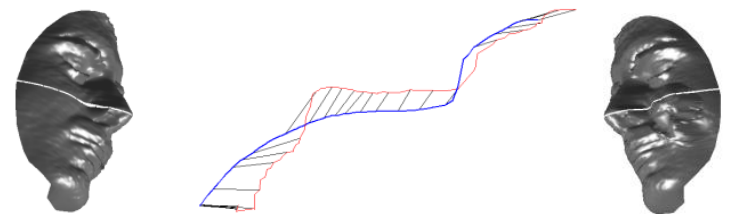

Fig. 4. An example of matching of symmetric radial curves of the face: a curve of the healthy half of the face (on the left) and a curve of swollen half (on the right).

$$
\left(O^{*}, \gamma^{*}\right)=\underset{(O, \gamma) \in S O(3) \times \Gamma}{\operatorname{argmin}} d_{c}\left(q_{1}, \sqrt{\dot{\gamma}} O\left(q_{2} \circ \gamma\right)\right)
$$

\section{EXPRIMENTAL RESULTS \& DISCUSSIONS}

To the best of our Knowledge, there is no database of 3D faces presenting tumors. We have selected 30 scans from FRAV 3D database (20 males, 10 females). Then, we have sculpted them to simulate left and right cheek tumefactions. In the following, we will provide a comparative performance of our preprocessing tasks with the state of the art solutions. Then, we will illustrate some examples of reconstructed cheeks based on the degradation of the facial tumor.

\subsection{Data Preprocessing}

In order to evaluate the performance of our algorithm, we have tested it on a PC with $2.2 \mathrm{GHz}$ Core 2 Duo processor with 2 GB memory. Table I presents the time consumed for different steps of our preprocessing algorithm and a comparison with state of the art. In these experiments, we have concluded that our algorithm is similarly efficient with the literature which has tested it on a PC with $2.39 \mathrm{GHz}$ Core 2 Duo processor with 3 GB memory [12].

Table 1. Comparison of computation costs

\begin{tabular}{|l|c|c|}
\hline \multirow{2}{*}{\multicolumn{1}{|c|}{ Step }} & \multicolumn{2}{c|}{ Time consumed (s) } \\
\cline { 2 - 3 } & Our algorithm & Drira et al [1,2] \\
\hline Fillig holes & 3.74 & 3.09 \\
\hline Nose tip detection & 0.078 & 0.091 \\
\hline Curves extraction & 1.68 & 1.6 \\
\hline
\end{tabular}

We have tested the nose tip detection algorithm on 60 scans of our swollen faces database. $69.6 \%$ of nose tips were detected compared with $99.1 \%$. This task may be influenced by the peaks left by the acquisition which changes the highest point of the face. To overcome this shortcoming, a smoothing filter should be applied to remove these peaks.

\subsection{Tumefaction degradation for face restoration}

We present the shape analysis of symmetrical radial curves. This task allows as calculating optimal deformations through geodesic paths between the corresponding symmetrical curves through both facial halves. The change in the standard deviation of the depth of the radial curves is a measure we have taken to locate the half that has the tumor. The variance is higher on the swollen halves. Then, we compare the 
deformed half with the healthy. Figure 5 shows examples of geodesic paths which illustrates the optimal deformation progressivity of the same persons.

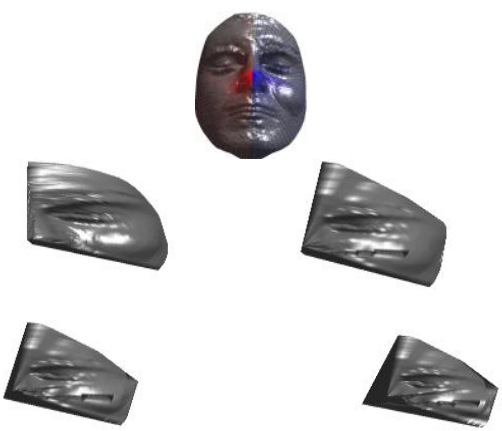

Fig. 5. Example of tumor progressivity.

\section{CONCLUSION \& PERSECTIVES}

In this work, we have presented a method for 3D face aided restoration that can be helpful for plastic surgery planning to predict and compare the outcome of such a procedure. The main goal is to help surgeons to restore cheek swollen faces. We have adopted a Riemannian analysis to calculate the geodesic path between the healthy half of the face and the swollen. We have followed up a series of 3D scan preprocessing tasks that allows to filling holes and extract radial curves which are selected symmetrically according to the vertical.

Additional outcomes are attempted in future. We will address more importance to the face tumors to be located all over the face and not to restrict them in unilateral cheek tumefactions. Also, we hope to develop an approach that extracts level curves to be coupled with radial curves for more performance.

The innovation of computer-aided plastic surgery lets this work to be more performed. Moreover, the tendency is to assist surgeons in planning a reconstructive and cosmetic surgery which can be implemented in computer aided plastic surgery real-time system.

\section{ACKNOWLEDGMENTS}

We thank PhD. Hassen Drira (University of Lille 1, LIFL) for many insightful and constructive comments. In particular, we thank, Mr. Zagrouba Ezzedine, Professor of computer science (Head of Research Team SIIVA - RIADI), for his valuable remarks.

\section{REFERENCES}

[1] M. R. Markiewicz and R. Bryan Bell, "The use of $3 D$ imaging tools in facial plastic surgery", facial plastic surgery Clin, vol.19, 2011, pp.655-682.

[2] A. Bottino, M. De Simone and A. Laurentini, "A computer-aided technique for planning plastic surgery based on 3D face scans: preliminary results", in Proc. of 1st Int. Conf. on 3D Body Scanning Technologies, Lugano, Switzerland, 2010, pp.213-221.

[3] Z. Kavagiuo, F. Bello, G. Scott, J. Hamann and D. Roberts, "Facial Plastic surgery planning using a $3 D$ surface deformation tool", Proceedings Medicine Meets Virtual Reality Conference MMVR, 2005, pp.247-250.

[4] S. M. Bhandarkar, A. S. Chowdhury, Yarong Tang, Jack $\mathrm{Yu}$, E.W. Tollner, "Surface matching algorithms for computer aided reconstructive plastic surgery", IEEE
International Symposium on Biomedical Imaging, Nano to Macro, Vol.1, April 2004, pp.740-743.

[5] T. Yee Lee, Y. N. Sun, Y. C. Lin, L. Lin and C. Lee, "Three-Dimensional facial model reconstruction and plastic surgery simulation", IEEE Transactions On Information Technology In Biomedicine, Vol. 3, $\mathrm{N}^{\mathrm{O}} .3$, September 1999, pp.214-220.

[6] V. Blanz and T. Vetter, "A morphable model for the synthesis of $3 D$ faces", $26^{\text {th }}$ annual conference on Computer graphics and interactive techniques, New York, USA, September 1999, pp.187-194.

[7] H. Drira, B. Ben Amor, M. Daouadi, and A. Strivastava, and R. Slama, "3D face recognition under expressions, occlusions and pose variation". IEEE Transactions on Pattern Analysis and Machine Intelligence, 2013, pp.2270-2283.

[8] H. Drira, B. Ben Amor, R. Slama, M. Daouadi and A. Strivastava, "Une nouvelle approche de reconnaissance de visages $3 D$ partiellement occultés", Reconnaissance des Formes et Intelligence Artificielle RFIA, Lyon, France, 2012, pp.978-986.

[9] A. Colombo, C. Cusano and R. Schettini, "ThreeDimensional occlusion detection and restoration of partially occluded faces", Journal of Math Imaging vision, Vol.3, Issue.3, December 2010, pp.105-119.

[10] A. Colombo, C. Cusano and R. Schettini, "Occlusions in Face Recognition: a 3D Approach", State of the art in face recognition, Julio Ponce and Adem Karahoca (Ed.), January 2009, pp.151-173.

[11] A. Colombo, C. Cusano and R. Schettini, "Gappy PCA classification for occlusion tolerant $3 D$ face detection", State of the Art in Face Recognition, Julio Ponce and Adem Karahoca (Ed.), January 2009, pp.151-173.

[12] H. Drira, B. Ben Amor, M. Daouadi, and A. Strivastava, "Pose and expression-invariant $3 D$ face recognition using elastic radial curves". British Machine Vision Conference BMVC, United Kingdom UK, 2010, pp.111.

[13] H. Drira, B. Ben Amor, L. Billihi, M. Daouadi, and A. Strivastava, "An experimental illustration of $3 D$ facial shape analysis under facial expressions", annals of telecommunications, 64 (5-6), Springer Verlag, Germany, 2009, pp.369-379.

[14] X. Chen, P. J. Flynn and K. W. Bowyer, "Fully automated facial symmetry axis detection in frontal color images", Automatic Identification Advanced Technologies, Fourth IEEE Workshop, 2005, pp.106111.

[15] J. Harguess and J. K. Aggarwal, "Is there a connection between face symmetry and face recognition?", IEEE Computer Society Conference on Computer Vision and Pattern Recognition, Department of ECE, June 2011, pp.66-73.

[16] L. Azinfar, M. Ravanfar and R. F. Rezai, "Computer aided ruler based on symmetric points detection for Botox injection", Journal of Medical Devices, Minneapolis, MN, September 2014, vol.8, Issue.3, pp.1131-1133. 
[17] A. Srivastava, E. Klassen, S. H. Joshi, and I. H. Jermyn, "Shape analysis of elastic curves in Euclidean spaces", IEEE Transactions on Pattern Analysis and Machine Intelligence, vol.37, Issue.7, 2011, pp.1415-1428.

[18] M. Daouadi, A. Strivastava and R. Veltkamp " $3 D$ face modeling, analysis and recognition", 224 pages, August 2013.

[19] S. H. Joshi, E. Klassen, A. Strivastava and I. Jermyn, " $A$ novel representation for Riemannian analysis of elastic curves in $R^{n}$," In: Proceeding of IEEE Computer Society Conference on Computer Vision and Pattern Recognition, June 2007, Minneapolis, MN. New York: IEEE. 2007, pp.1-7.

[20] M. D. Levine and Y. Yu, "State-of-the-art of 3D facial reconstruction methods for face recognition based on a single $2 D$ training image per person ", Pattern Recognition Letters, July 2009, Vol. 30, Issue 10, pp. 908-913.

[21] I. Kemelmacher-Shlizerman and R. Basri, "3D Face reconstruction from a single image using a single reference face shape", IEEE Transactions on Pattern Analysis and Machine Intelligence (PAMI), February 2011, vol.33, Issue.2, pp.394-405.

[22] W. Ben Soltana, M. Ardabilian, P. Lemai, H. Di, P. Szeptycki, L. Chen, B. Ben Amor, H. Drira, M. Daoudi,
N. Erdogmus, L. Daniel, J. L. Dugelay and J. Colineau, "3D face recognition: a robust multi-matcher approach to data degradations", $5^{\text {th }}$ IAPR International Conference on Biometrics Compendium, IEEE, New Delhi, April 2012, pp.103-110.

[23] H. Drira, B. Ben Amor, M. Daouadi, and A. Strivastava, "A Riemannian analysis of $3 D$ nose shapes for partial human biometrics", IEEE 12th International Conference on Computer Vision, Kyoto, 2009, pp.2050-2057

[24] S. Prabahar and K. Usha Kingsly Devi, "An optimistic approach for plastic surgery face recognition", Journal of Research in Electrical and Electronics Engineering (ISTP-JREEE), Vol.3, Issue.3, May 2014, pp.38-43.

[25] E. Keeve, S. Girod, R. Kikinis, and B. Girod "Deformable modeling of facial tissue for craniofacial surgery simulation", Official journal of the International Society for Computer Aided Surgery, Vol.3, 1998, pp.228-194.

[26] H. Drira, B. Ben Amor, M. Daoudi, and A. Srivastava, "Nasal region contribution in $3 D$ face biometrics using shape analysis framework", Third International Conference, ICB 2009, Vol.5558, Italy, June 2009, pp.357-366. 